\title{
Neglected tropical diseases: elimination and eradication
}

\author{
Authors: Christopher Bodimeade, ${ }^{A}$ Michael Marks ${ }^{B}$ and David Mabey ${ }^{C}$
}

The term neglected tropical diseases (NTDs) describes a disparate group of diseases which affect populations living in poverty and are important causes of morbidity and mortality worldwide. Global programmes for the control of NTDs benefit large-scale donations made by pharmaceutical companies. A number of NTDs have internationally agreed targets for their control, elimination and eradication. Eradication is defined as the permanent reduction to zero of the worldwide incidence of infection. Elimination is defined as the reduction to zero of the incidence of infection in a specified geographic area. Considerable progress has been made towards elimination and eradication of some NTDs but unexpected new challenges have emerged which threaten the eventual achievement of these goals.

\section{Neglected tropical diseases}

In the early 2000s the term neglected tropical diseases (NTDs) emerged to describe a disparate group of diseases which disproportionately affect populations living in poverty; cause important morbidity and mortality - including stigma and discrimination - in such populations, justifying a global response; are amenable to broad control, elimination or eradication through mass treatment, intensified or innovative case management, vector control or veterinary public health; and are relatively neglected by research. ${ }^{1,2}$ The World Health Organization (WHO) currently recognises 20 diseases which meet these criteria (Table 1).

NTD programmes benefit from some of the largest pharmaceutical donation programmes ever made. Several pharmaceutical companies agreed to donate unlimited supplies of medication for as long as it was needed to support global programmes. These donations, supported by substantial funds for implementation from partners, including the UK Department for International Development, have allowed substantial progress to be made in reducing morbidity from these conditions. ${ }^{3}$ Despite this progress, considerable challenges remain in the control, elimination and eradication of NTDs. In this article we outline the principles of disease elimination and eradication as they apply to NTD programmes and illustrate some of the challenges in achieving the aim of disease eradication.

Authors: Amedical student, University of Leicester, Leicester, UK; ${ }^{\text {B }}$ assistant professor, London School of Hygiene and Tropical Medicine, London, UK; C professor of communicable diseases, London School of Hygiene and Tropical Medicine, London, UK

\section{Principles of elimination and eradication}

A common feature of many of the NTDs is the existence of international targets for their control, elimination and eradication (see Table 1). These terms are often used interchangeably and sound similar but in fact have specific definitions. The terms were first well defined at the Dahlem Workshop in 1997. ${ }^{4}$

Eradication is defined as the permanent reduction to zero of the worldwide incidence of infection. Elimination is defined as the reduction to zero of the incidence of infection in a specified geographic area. These definitions also highlight other important points. Firstly, eradication is by definition a global term - a disease cannot be eradicated at the national level. Secondly, even following elimination of a disease, ongoing control efforts are required to avoid re-introduction. For example, polio has been eliminated in the Americas but vaccination is still required until the goal of global eradication is achieved.

The Dahlem Workshop also highlighted criteria to be considered when assessing the feasibility of disease eradication. These criteria consist of both biological and political factors and are outlined in Table 2.

\section{Key points}

There are 20 conditions currently recognised by the World Health Organization as neglected tropical diseases

Ten of these diseases are targeted for either elimination or eradication

Considerable progress has been made towards guinea worm eradication but the occurrence of disease in dogs is a major challenge to its eventual eradication

Mass treatment with azithromycin is a promising strategy for the eradication of yaws but drug resistance is a significant concern

Progress has been made towards the control, elimination and eradication of many neglected tropical diseases supported by large scale drug donations

KEYWORDS: Neglected tropical diseases, guinea worm, yaws, trachoma, lymphatic filariasis 
Table 1. The World Health Organization neglected tropical diseases

\section{Neglected tropical diseases}

Schistosomiasis

Onchocerciasis

Lymphatic filariasis

Trachoma

Yaws

Soil transmitted helminths

Guinea worm

African trypanosomiasis

Visceral leishmaniasis

Leprosy

Taeniasis/cysticercosis

Echinococcosis

Foodborne trematodes

Chagas disease

Buruli ulcer

Rabies

Dengue and chikungunya

Mycetoma

Scabies

MDA = mass drug administration.

\section{Control strategy Elimination target}

MDA (praziquantel), Yes (in China) sanitation, snail control

MDA (ivermectin), Yes (in vector control Americas)

MDA (ivermectin, Yes albendazole), vector control

MDA (azithromycin), Yes water, sanitation, education

MDA Yes (azithromycin) eradication MDA (albendazole)

Safe water, health education

Case finding and treatment, vector control

Case finding and treatment

Case finding and treatment

Sanitation,

No

meat inspection, vaccination of pigs

Abbattoir control,

No treatment of dogs, education

Treatment of sheep, health education

Vector control, blood screening

Case finding and countries) treatment

Vaccination of dogs, health education

Vector control

No

Case finding and No treatment

Not yet defined. No Possibly MDA

(ivermectin)

Not yet defined
Table 2: Criteria and requirements for disease

elimination and eradication

\section{Biological criteria}

Availability of an effective intervention ie a drug or vaccine.

Diagnostic test with adequate sensitivity and specificity.

Absence of an environmental or animal reservoir.

\section{Social and political criteria}

Disease must be recognised to be of public health importance.

Eradication must be widely perceived to be a worthy goal.

Technically feasible intervention must be found to be effective in a geographical region.

Political commitment must be gained at the highest level including potentially a World Health Assembly resolution.

There must be an advocacy national level. plan for the global, regional and

A number of NTDs have neither an 'eradication' nor an 'elimination' target. Instead they have a target of 'elimination as a public health problem'. This term is not well defined but broadly can be considered to mean reduction in the prevalence and/or incidence of a disease to such a level that it no longer contributes significantly to morbidity (or mortality) at the population level. The closeness of the terms 'elimination as a public health problem' and 'elimination' risks creating confusion in the minds of policymakers for whom the terms may appear to be analogous. This risk is compounded by the absence of a standard definition for 'elimination as a public health problem' - instead the definitions used vary between disease programmes. The targets for each disease have also tended to shift over time most commonly from less challenging (elimination) to more challenging (eradication) targets.

\section{The London Declaration}

Individual targets for neglected tropical disease eradication and elimination have emerged over a period of years. In 2012, WHO published a roadmap for neglected tropical diseases ${ }^{5}$ and this was followed by a landmark meeting in London which culminated in the 'London Declaration' for neglected tropical diseases. This meeting bought together policymakers, pharmaceutical companies, non-governmental organisations and academics to agree a common set of disease targets and priorities.

\section{Progress and challenges - case studies in NTD eradication}

\section{Guinea worm}

Guinea worm disease is caused by infection with Dracunculus medinensis. Infection occurs when humans ingest contaminated water containing cyclops (microscopic crustaceans) which act as vectors for the Guinea worm larvae. Once in the digestive tract, larvae escape from the cyclops and penetrate the subcutaneous soft tissues where they mate. Over the following year, the female 
worms migrate to the legs and feet where a small painful blister forms and the worm emerges. It then releases its larvae into water sources allowing the cycle of infection to repeat.

In the 1980s, the control and eventual elimination of Guinea worm were adopted as targets by the World Health Assembly (WHA) as part of the United Nation's International Drinking Water Supply and Sanitation decade. ${ }^{6}$ In 1991, the WHA formally committed to global eradication of Guinea worm. ${ }^{7}$

The strategy for eradication is based primarily on behaviour change. Health education, combined with filter distribution, vector control, safe water provision and case containment, enables affected communities to break the cycle of transmission. ${ }^{8}$ These methods, spearheaded by the Carter Center, have had considerable success. In 1986, there were an estimated 3.5 million cases in 20 countries; ${ }^{9}$ in 2017 only 30 cases of Guinea worm were reported in two countries, Ethiopia and Chad. ${ }^{10}$

Despite the progress, significant challenges remain. Continuing civil war in South Sudan has disrupted surveillance activities. Following 19 consecutive months without a case being reported, three have been detected there since May $2018 .{ }^{11}$ Chad remains a reservoir of the disease largely due to the significant number of cases in dogs, 830 of which were detected in $2017 .{ }^{10}$ The hypothesised model of transmission in dogs involves consumption of Guinea worm larvae contained in the fresh entrails of fish. ${ }^{12}$ Finally, the recent discovery of a human case of Guinea worm in Angola, a country that has never previously documented a case of the disease, serves as a reminder of the surprises that eradication programmes can produce. ${ }^{11}$ The source of the worm, identified in April 2018, remains a mystery and extensive investigations are ongoing. These developments call the imminent success of the eradication programme into question.

\section{Yaws}

Yaws, caused by Treponema pallidum subsp pertenue, is one of two NTDs currently targeted for global eradication. Yaws is closely related to syphilis but predominantly affects children living in remote tropical communities. If untreated the disease may cause destructive and disfiguring lesions of the skin, bones and soft tissues. During the mid-twentieth century the disease was widespread throughout the tropics and was considered a major public health problem. 13,14 $^{2}$

In 1949, the WHA passed a resolution supporting efforts for the control and elimination of yaws. Between 1952 and 1962, WHO and the United Nations International Children's Emergency Fund led a joint global campaign to eradicate yaws. ${ }^{14}$ More than 300 million individuals were assessed as part of the campaign and more than 50 million treated, a monumental effort at the time. However, these efforts were ultimately not successful. Whilst the programme did significantly reduce the global prevalence of yaws, by as much as $98 \%$, the incidence of the disease rebounded in a number of countries in the 1970s. Interest in yaws eradication then waned and the disease fell off the global health agenda. Interest in yaws eradication was restarted by a landmark study conducted in Papua New Guinea which demonstrated that a single oral dose of azithromycin was non-inferior to intramuscular benzathine benzylpenicillin. ${ }^{15}$

Mass drug administration (MDA) with azithromycin has been widely used as part of the WHO strategy for the elimination of trachoma as a public health problem. ${ }^{16}$ Given the evidence of the safety of MDA with azithromycin and the efficacy of azithromycin for the treatment of yaws, WHO developed a revised strategy for the eradication of yaws (the Morges strategy). This strategy emphasises an initial round of MDA using azithromycin in endemic communities. Following this it may be appropriate to conduct further MDA or to switch to a strategy of treating active cases and their contacts (total targeted treatment). ${ }^{17}$ Initial assessments of the efficacy of azithromycin MDA were conducted in both the Pacific and west Africa and demonstrated considerable impact of MDA on the prevalence of yaws. However, despite these initial successes, interruption of transmission was not achieved. ${ }^{18-20}$ Furthermore resistance to azithromycin emerged in the pilot study in Papua New Guinea. Although subsequent treatment with benzathine benzylpenicillin was used to successfully ring-fence this outbreak, these data highlight the risk of emerging azithromycin resistance threatening yaws eradication efforts. ${ }^{21}$

Finally, there is also evidence that non-human primates are also infected with Treponema pallidum subsp pertenue. ${ }^{22,23}$ While no evidence currently exists for zoonotic transmission, the existence of a possible animal reservoir highlights the challenges that may emerge during an eradication campaign.

\section{Conclusions}

Enormous progress has been made towards the control, elimination and eradication of neglected tropical diseases. These achievements have been based on unprecedented levels of support from pharmaceutical companies, governments and private donors. Despite this, challenges remain towards the eventual attainment of the London Declaration 2020 targets and the eventual elimination and eradication of these diseases remains uncertain.

\section{References:}

1 Hotez PJ, Fenwick A, Savioli L, Molyneux DH. Rescuing the bottom billion through control of neglected tropical diseases. Lancet 2009:373:1570-5

2 Hotez PJ, Alvarado M, Basáñez M-G et al. The global burden of disease study 2010: interpretation and implications for the neglected tropical diseases. PLoS Negl Trop Dis 2014;8:e2865.

3 Hotez PJ, Fenwick A, Ray SE, Hay SI, Molyneux DH. 'Rapid impact' 10 years after: The first 'decade' (2006-2016) of integrated neglected tropical disease control. PLoS Negl Trop Dis 2018;12:e0006137.

4 Dowdle WR. The principles of disease elimination and eradication. Bull World Health Organ 1998;76:22-5.

5 World Health Organization. Accelerating work to overcome the global impact of neglected tropical dieases - a roadmap for implementation. Geneva: WHO, 2012. www.who.int/neglected_diseases/ NTD_RoadMap_2012_Fullversion.pdf.

6 World Health Organization. World Health Assembly Resolution 34.25: International drinking water supply and sanitation decade. WHO, 1981. www.who.int/neglected_diseases/mediacentre/ WHA_34.25_Eng.pdf?ua=1.

7 World Health Organization. World Health Assembly Resolution 42.29: Eradication of dracunculiasis. WHO, 1989. www.who.int/ neglected_diseases/mediacentre/WHA_42.29_Eng.pdf?ua=1.

8 Biswas G, Sankara DP, Agua-Agum J, Maiga A. Dracunculiasis (Guinea worm disease): eradication without a drug or a vaccine. Philos Trans R Soc B Biol Sci 2013;368:20120146.

9 Watts SJ. Dracunculiasis in Africa in 1986: Its geographic extent, incidence, and at-risk population. Am J Trop Med Hyg 1987:37:119-25. 
10 World Health Organization Collaborating Center for Dracunculiasis Eradication. Guinea worm wrap-up \#253. Atlanta: The Carter Center, 2018. www.cartercenter.org/resources/pdfs/news/health_ publications/guinea_worm/wrap-up/253.pdf

11 World Health Organization Collaborating Center for Dracunculiasis Eradication. Guinea worm wrap-up \#256. Atlanta: The Carter Center, 2018.www.cartercenter.org/resources/pdfs/news/health_ publications/guinea_worm/wrap-up/256.pdf

12 Sreenivasan N, Weiss A, Djiatsa J-P et al. Recurrence of Guinea worm disease in Chad after a 10-year absence: risk factors for human cases identified in 2010-2011. Am J Trop Med Hyg 2017:97:575-82.

13 Urrutia RP, Merisier D, Small M et al. Unmet health needs identified by Haitian women as priorities for attention: a qualitative study. Reprod Health Matters 2012;20:93-103.

14 Asiedu K, Amouzou B, Dhariwal A et al. Yaws eradication: past efforts and future perspectives. Bull World Health Organ 2008;86:499-499A.

15 Mitjà $\mathrm{O}$, Hays R, Ipai A et al. Single-dose azithromycin versus benzathine benzylpenicillin for treatment of yaws in children in Papua New Guinea: an open-label, non-inferiority, randomised trial. Lancet 2012;379:342-7.

16 Emerson PM, Burton MJ, Solomon AW, Bailey R, Mabey DC. The SAFE strategy for trachoma control: using operational research for policy, and implementation. Bull World Health Organ 2006;84:613-9.
17 The World Health Organization. Eradication of yaws - the Morges Strategy. Wkly Epidemiol Rec 2012;87:189-194.

18 Abdulai AA, Agana-Nsiire P, Biney F et al. Community-based mass treatment with azithromycin for the elimination of yaws in Ghana - Results of a pilot study. PLoS Negl Trop Dis 2018;12:e0006303.

19 Marks M, Vahi V, Sokana O et al. Impact of community mass treatment with azithromycin for trachoma elimination on the prevalence of yaws. PLoS Negl Trop Dis 2015;9:e0003988.

20 Mitjà $\mathrm{O}$, Houinei W, Moses $\mathrm{P}$ et al. Mass treatment with single-dose azithromycin for yaws. N Engl J Med 2015;372:703-10.

21 Mitjà $\mathrm{O}$, Godornes $\mathrm{C}$, Houinei W et al. Re-emergence of yaws after single mass azithromycin treatment followed by targeted treatment: a longitudinal study. Lancet 2018;391:1599-1607.

22 Knauf S, Gogarten JF, Schuenemann V] et al. Nonhuman primates across sub-Saharan Africa are infected with the yaws bacterium Treponema pallidum subsp pertenue. Emerg Microbes Infect 2018;7:157.

23 Chuma IS, Batamuzi EK, Collins DA et al. Widespread Treponema pallidum infection in nonhuman primates, Tanzania. Emerg Infect Dis 2018;24:1002-9.

Address for correspondence: Dr Michael Marks, London School of Hygiene and Tropical Medicine, Keppel Street, London WC1E 7HT, UK.

Email: michael.marks@Ishtm.ac.uk

\section{NGC Natasea

\section{New to systematic reviewing, or need to improve your critical appraisal skills?}

Our intensive 1-day courses will introduce you to the key principles of critical appraisal and systematic reviewing, and equip you with the basic skills to put your knowledge into practice.

$>$ Introduction to critical appraisal

> Systematic review and meta-analysis in action

Discount available if both courses are booked together.

'Informative and practical'

Dr Rajeswari Ramaraj
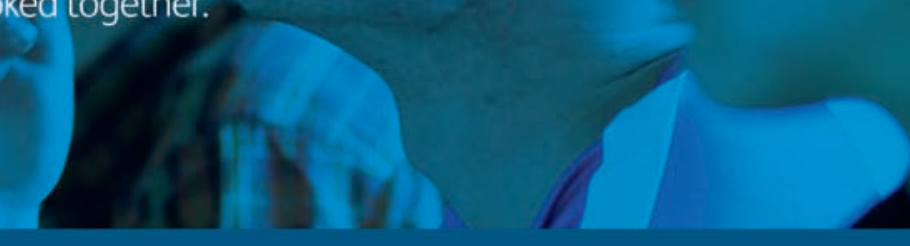

For course dates and more information visit: www.rcplondon.ac.uk/ngc-training-courses 